\title{
A Nal-based cryogenic scintillating calorimeter: status and results of the COSINUS project
}

\author{
N. Di Marco*广, S. Pirro \\ INFN - Laboratori Nazionali del Gran Sasso, I-67010 Assergi - Italy
}

G. Angloher, M. Mancuso, F. Petricca, F. Pröbst

Max-Planck-Institut für Physik, D-80805 München - Germany

P. Carniti, L. Gironi, C. Gotti, L. Pagnanini, G. Pessina

INFN - Sezione di Milano Bicocca, I-20126 Milano - Italy and Dipartimento di Fisica,

Università di Milano-Bicocca, I-20126 Milano - Italy

\section{F. Reindl, J. Schieck, C. Schwertner, M. Stahlberg}

Institut für Hochenergiephysik der ÖAW, A-1050 Wien - Austria and Atominstitut, Technical University Vienna, A-1020 Wien - Austria

\section{K. Schäffner, V. Zema}

Gran Sasso Science Institute, I-67100 L'Aquila - Italy and INFN - Laboratori Nazionali del Gran Sasso, I-67010 Assergi - Italy

\section{Y. Zhu}

Shanghai Institute of Ceramics, Chinese Academy of Sciences

\begin{abstract}
The COSINUS (Cryogenic Observatory for SIgnals seen in Next-generation Underground Searches) project aims to provide a model-independent cross-check of the long-standing DAMA/LIBRA claim on the observation of dark matter. The use of sodium iodide (NaI) crystals, operated at cryogenic temperature as scintillating calorimeters, offers both a low energy threshold for nuclear recoils and the possibility to perform particle discrimination. Indeed, the dual read-out of phonon and light allows to perform signal-to-background discrimination on an eventby-event basis, a unique feature in comparison to other NaI-based dark matter searches. In this paper we will present the COSINUS detector concept together with status and future prospects of the project.
\end{abstract}

Neutrino Oscillation Workshop (NOW2018)

9 - 16 September, 2018

Rosa Marina (Ostuni, Brindisi, Italy)

\footnotetext{
*Speaker.

${ }^{\dagger}$ natalia.dimarco@lngs.infn.it
} 


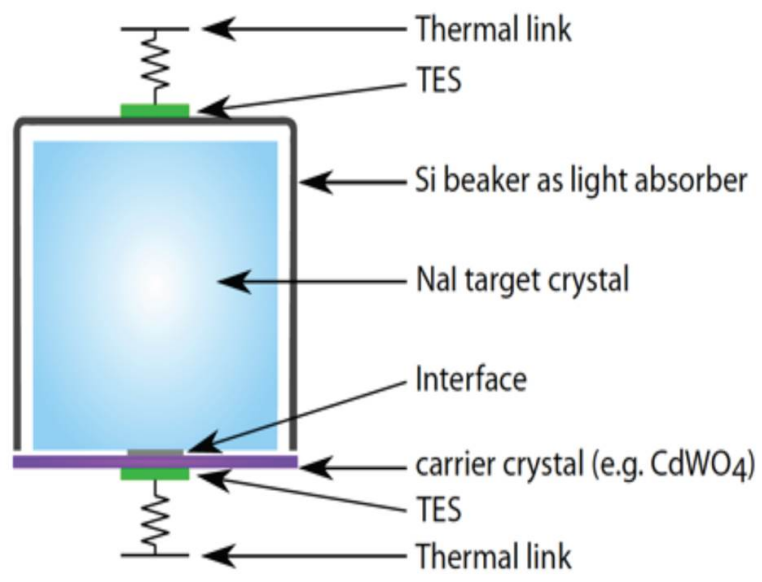

Figure 1: Cosinus detector module consisting of two independent channels: a NaI crystal that is supported and read out via a carrier crystal and a separated beaker-shaped light detector. Both detectors are equipped with highly sensitive thermometers, so-called TES (Transition Edge Sensors) to detect the tiny temperature variations due to particle interactions in the $\mathrm{NaI}$.

\section{Introduction}

Worldwide experimental efforts in the field of Dark Matter (DM) search are strongly motivated by the well-established evidence for the existence of DM based on astrophysical observations. Direct search techniques essentially rely on the assumption of massive DM particles, with a mass in the $(\mathrm{GeV}-\mathrm{TeV})$ range and interaction strength of the scale of the weak processes (the so called WIMPs), scattering off a target nuclei in a earth-bound detector. The experimental approaches are based on single or dual channel read-out with detection of scintillation light, phonon and/or charge signals. The analysis is related on the detection of an excess of events over the expected background or on the detection of an annual modulation of the event rate caused by the seasonal variation of the Earth's velocity with respect to the dark matter halo.

The statistically robust modulation signal $(12.9 \sigma)$ [ [ $[\mathbf{W}]$ with phase and period matching the expectation for DM interaction, reported by the DAMA/LIBRA Collaboration is apparently in contrast with the null results of the other direct search experiments. Nonetheless, the comparison of the results of different experiments necessarily relies on model-assumptions such as the DM velocity distribution and the interaction mechanism. Therefore, only an experiment using the same target material as DAMA/LIBRA, would be immune to material-dependencies. Among the different R\&D projects and experiments employing NaI crystals as target, the COSINUS project is the only one featuring a dual channel read-out allowing to perform particle identification.

\section{NaI-based cryogenic scintillating calorimeter}

The COSINUS project [3] is based on the use of sodium iodide crystals (NaI) operated at cryogenic temperature. A particle interacting in a cryogenic scintillating calorimeter produces 
two coincident signals: the major part of the deposited energy is converted into phonons (heat signal) while a small fraction $(\mathrm{O}(\%))$ is emitted in form of scintillation light. The heat signal is exploited to precisely measure the energy deposited in the target and is almost independent from the particle type. The amount of scintillation light, instead, strongly depends on the particle type. As a consequence, the light to phonon ratio, the so-called light yield (LY), is characteristic for each type of event and can be used to perform particle identification, thus allowing the suppression of the dominant $\beta / \gamma$ background with respect to nuclear recoil events. $\beta / \gamma$ particles produce most light and, by definition, get assigned a LY $=1$, while $\alpha$-particles and nuclear recoils show a lower LY quantified by the so-called quenching factors (QFs).

In a typical COSINUS detector module, the phonon signal is read-out by a Transition Edge temperature Sensor (TES) consisting of a superconducting thin film and evaporated on a crystal, called carrier (e.g. $\mathrm{CdWO}_{4}$ ), coupled with the $\mathrm{NaI}$ target via an interface (typically silicon oil). The use of the carrier crystal is needed in order to decouple the fragile NaI-crystal from the TES production procedure. The small temperature rise $(\mathrm{O}(10) \mu \mathrm{K})$ caused by a particle interaction results in a change in the resistance of the TES which can be measured using sensitive amplifiers based on Superconducting Quantum Interference Devices (SQUIDs). The NaI crystal is then inserted in a beaker-shaped light detector made from high-purity silicon of about $\sim 40 \mathrm{~mm}$ in diameter and height, and $<400 \mu \mathrm{m}$ of wall-thickness. The light detector is equipped with a second TES reading the temperature rise of the beaker caused by the absorption of scintillation photons (see Fig W). Thanks to the large fraction of the NaI crystal surface coverage, the light detector features a very high light collection efficiency while simultaneously serving as an active veto for external backgrounds and radioactive decays close to the surfaces of the NaI crystal and its surrounding material.

\section{Status, results and future prospects of the COSINUS project}

Already with the first COSINUS prototype we confirmed the feasibility of building a NaIbased cryogenic scintillating calorimeter by observing a linear relation between the light output and the energy deposited in the NaI crystal [四].

With the second prototype we implemented the full COSINUS detector design with a NaI crystal housed in a Si-beaker-shape light absorber [5]. Figure \ shows data of the second prototype in the light yield vs. energy plane. Two main event distributions appear: the events due to $\beta / \gamma$ interactions in $\mathrm{NaI}$ at a light yield value around one including calibration lines from an external

${ }^{241} \mathrm{Am} \gamma$-source and an event distribution due to interactions in the carrier at a light yield of zero, since, in this case, there is not an associated light signal in the light detector. The regions where interactions due to $\mathrm{Na}$ (blue) and I (green) recoils are expected are calculated assuming quenching factors taken from [6]. In this measurement, we achieved a (hardware) energy threshold for the $\mathrm{NaI}$ of $\left(8.26 \pm 0.02\right.$ (stat.)) $\mathrm{keV}$ and the width of the $60 \mathrm{keV}{ }^{241} \mathrm{Am}$ peak is found to be (4.508 \pm 0.064 (stat.)) $\mathrm{keV}$. For the light detector a baseline energy resolution (in absolute energy scales) of $\sigma=15 \mathrm{eV}$ is determined and the fraction of scintillation light with respect to the total deposited energy in the $\mathrm{NaI}$ crystal is found to be $13 \%$. Moreover, the light detector shows an energy threshold of $\sim 0.6$ keVee exceeding the performances of PMTs coupled with $\mathrm{NaI}(\mathrm{Tl})$ at room temperatures $(\sim 1 \mathrm{keVee})$. In both measurements, we found that $\mathrm{NaI}$ exhibits a different pulse shape compared 


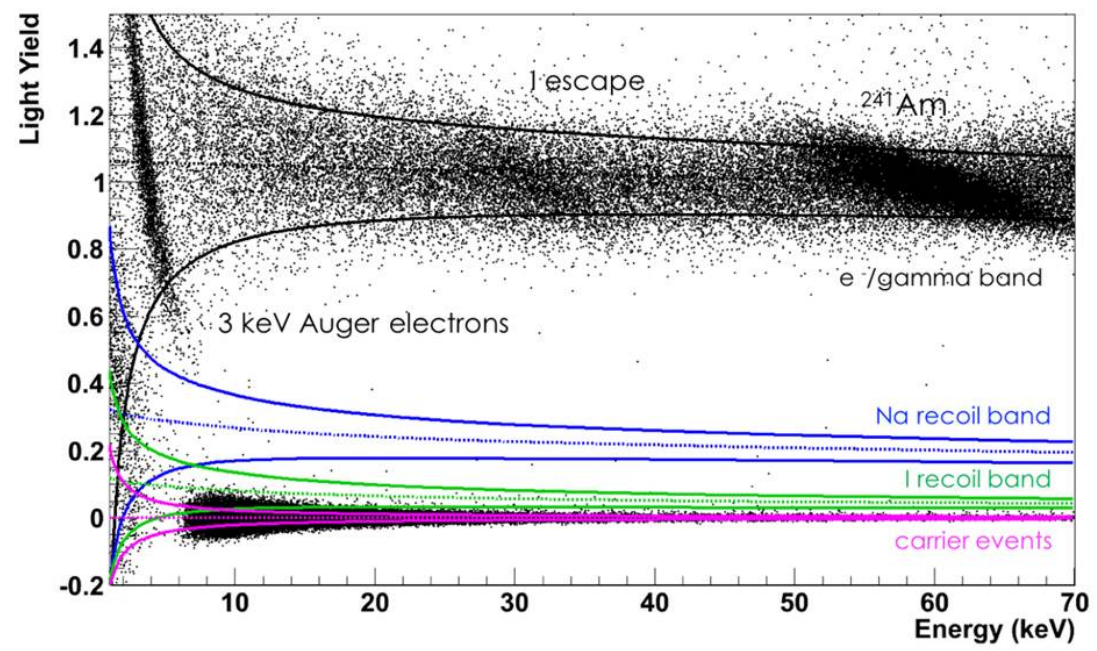

Figure 2: Calibration data in the light yield vs. energy plane for a detector consisting of a $\sim 70 \mathrm{~g}$ NaI crystal and beaker-shaped Si light absorber. The bands correspond to the following event classes: electron recoils in black, scatterings off $\mathrm{Na}$ and I in blue and green. The band at zero light yield is the so-called carrier band (magenta) due to interactions taking place in the carrier crystal itself.

to other materials and cannot be described by the commonly two-component model [ [U] used for cryogenic particle detectors, being, a third (thermal) component, needed to properly fit the pulses. As a result of this analysis we can conclude that the peculiarity of $\mathrm{NaI}$ is a long decay time of the pulse [8, 团].

At present, several other prototypes are under test including new batches of highly radiopure crystals produced in collaboration with the SICCAS using different concentration of Tl doping, improving the TES/crystal coupling and testing new TES concepts. The data daking is currently ongoing and new results are expected soon. Moreover, a neutron test beam at the Tandem accelerator of the Maier-Leibnitz-Laboratorium of TUM and LMU in Munich, to directly measure the QFs of a NaI crystal operated at cryogenic temperatures, has been recently concluded.

In order to move from the current $R \& D$ phase to the realization of the real experiment it is necessary to design and build a low-background cryogenic facility. Therefore, in parallel with measurements and hardware developments, we are carrying out a complete Monte Carlo simulation of the detector shield against the external radioactivity and we are studying the whole design of the experimental apparatus. The commissioning of the COSINUS experiment is foreseen in 2020. For a detailed study of COSINUS physics reach the reader is addressed to [ए]]]. 


\section{References}

[1] R. Bernabei et al., Universe 4 (2018) no.11, 116 doi:10.3390/universe4110116

[2] R. Bernabei et al., [arXiv:1805.10486 [hep-ex]].

[3] G. Angloher et al., Eur. Phys. J. C 76 (2016) no.8, 441 doi:10.1140/epjc/s10052-016-4278-3 [arXiv:1603.02214 [physics.ins-det]].

[4] G. Angloher et al. [COSINUS Collaboration], JINST 12 (2017) no.11, P11007 doi:10.1088/1748-0221/12/11/P11007 [arXiv:1705.11028 [physics.ins-det]].

[5] K. Schäffner et al., J. Low. Temp. Phys. 193 (2018) no.5-6, 1174. doi:10.1007/s10909-018-1967-3

[6] V. I. Tretyak, Astropart. Phys. 33 (2010) 40 doi:10.1016/j.astropartphys.2009.11.002 [arXiv:0911.3041 [nucl-ex]].

[7] F. Pröbst, Journal of Low Temperature Physics 100 (1995) doi:10.1007/BF00753837

[8] F. Reindl et al. [COSINUS Collaboration], arXiv:1711.01482 [astro-ph.IM].

[9] N. Di Marco et al., J. Phys. Conf. Ser. 1056 (2018) no.1, 012017. doi:10.1088/1742-6596/1056/1/012017

[10] F. Kahlhoefer, F. Reindl, K. Schäffner, K. Schmidt-Hoberg and S. Wild, JCAP 1805 (2018) no.05, 074 doi:10.1088/1475-7516/2018/05/074 [arXiv:1802.10175 [hep-ph]]. 